\title{
Cultures multiples de plants d'épinette noire sous serre et ombrière
}

\author{
par
}

Ronald M. Girouard'

\section{Résumé}

Ce travail présente les résultats d'une étude réalisée sur des plants d'épinette noire (Picea mariana [Mill.] B.S.P.), dont le but était de déterminer si, à l'aide d'une serre chauffée le moins longtemps possible et d'une ombrière, des cultures multiples sont réalisables tout en respectant les critères d'acceptation des plants du ministere de l'Énergie et des Ressources du Québec. La réponse est oui, mais seulement pour deux cultures par année à Sainte-Foy: une d'hiver, l'autre d'été. Celle d'hiver peut être ensemencée au début de février et les plants sortis de serre vers le 25 mai pour être acclimatés sous ombrière. Celle d'été peut être ensemencée le 25 mai et les plants mis sous ombrière au plus tard le 17 août.

Mots clés: Picea mariana (Mill.) B.S.P., cultures multiples, serre, ombrière, tube québécois.

\begin{abstract}
Black spruce (Picea mariana [Mill.] B.S.P.) seedlings were studied to determine whether multiple crops that meet the Quebec's Department of Energy and Resources standards, can be produced in a greenhouse, heated no longer than necessary, and a shadehouse. Only two crops a year are possible at Sainte-Foy: one in winter and the other in summer. The winter crop can be sown in early February and the plants taken out of the greenhouse close to May 25 for acclimatization in a shadehouse. The summer crop can be sown on May 25 and the plants placed in a shadehouse no later than August 17.
\end{abstract}

Key words: Picea mariana (Mill.) B.S.P., multiple crops, greenhouse, shadehouse, Quebec tube.

\section{Introduction}

Vers la fin des années soixante-dix, l'idée de produire à grande échelle des cultures multiples de plants forestiers était très répandue dans la province de Québec. Les travaux publiés par Schabas (1978) et Paillé et Brown (1980) sur les cultures mutliples d'épinette noire et Kay (1973), Owston (1974) et Myers (1979) sur d'autres essences étaient connus.

Peu de temps après, Carlson (1983) et McNeish et Heinstein (1983) ont décrit des calendriers de production de trois cultures d'épinette noire. Zalasky (1983) a fait mention d'un calendrier de trois à six cultures ayant une durée sous serre de quatre à neuf semaines. Ce calendrier très chargé n'a pas servi longtemps puisque l'endurcissement au gel d'un fort pourcentage de plants était incomplet (S. Navratil, communication personnelle, 1985).

Dans les pépinières où l'emphase est mise sur deux cultures par année, une d'hiver et une d'été, ce sont parfois des plants d'épinette noire qui sont ensemencés deux fois (Schabas 1978, Kelly 1982, Carlson 1983, Deschênes et Whalen 1983, Langlois 1985). Dans d'autres cas, c'est la culture d'épinette noire en hiver et la culture de pin gris en été

Centre de foresterie des Laurentides, Service canadien des forêts, 1055, rue du P.E.P.S., C.P. 3800, Sainte-Foy (Québec), G1V 4C7
(Forcier 1980, Léger 1980, Barteaux et Kreiberg 1982, Clark 1983).

Ce rapport présente les résultats d'une étude réalisée à Sainte-Foy, Québec, en 1981 dont le but était de déterminer si, à l'aide d'une serre chauffée le moins longtemps possible et d'une ombrière, des cultures multiples peuvent être produites tout en respectant les critères d'acceptation des plants du ministère de l'Énergie et des Ressources (MER) du Québec.

\section{Matériel et méthodes}

\section{Matériel végétal et conteneur}

Des plants d'épinette noire (Picea mariana [Mill.] B.S.P.), en provenance de Damville, comté de Roberval, Québec $\left(46^{\circ} 04^{\prime}\right.$ de latitude nord, $73^{\circ} 00^{\prime}$ de longitude ouest, $335 \mathrm{~m}$ d'altitude), furent cultivés sur tourbe dans des tubes québécois. Ce type de conteneur était cylindrique, avait un diamètre de $4 \mathrm{~cm}$, une longueur de $10 \mathrm{~cm}$ et un volume de $126 \mathrm{~cm}^{3}$. Sa paroi était constituée d'une feuille de papier cellukraft blanc opaque liée, d'un côté seulement, à une mince couche de polyéthylène (papier:polyéthylène 18:10). La feuille composée était roulée et collée en longueur pour former un tube avec le polyéthylène du côté interne (Girouard 1985). Les tubes, en provenance du Centre de recherche 
industrielle du Québec (CRIQ), contenaient de la tourbe Pointe-au-Père dont la densité était $0,1 \mathrm{~g} / \mathrm{cm}^{3}$ (Barbulescu 1982, Gonzalez 1981).

\section{Conduite de l'expérience}

L'expérience a débuté le 9 février 1981 sous serre en verre à Sainte-Foy, Québec $\left(46^{\circ} 47^{\prime}\right.$ de latitude nord, $71^{\circ} 18^{\prime}$ de longitude ouest, $62 \mathrm{~m}$ d'altitude). Des ensemencements ont eu lieu aux trois semaines jusqu'au 15 juin (tableau 1). A partir du 25 mai, pour les plants âgés d'au moins six semaines, deux plateaux (90 plants de chaque date d'ensemencement) furent mis sous ombrière à des intervalles de trois semaines. Le 28 septembre, l'âge des plants de 43 traitements variait de 15 à 33 semaines. Chaque traitement était en soi un cycle serre-ombrière exprimé en semaines.

Les conditions de culture sous serre étaient généralement celles décrites par D'Aoust et Trudel (1985) et qu'on peut résumer comme suit: température minimale de $22^{\circ} \mathrm{C}$ (jour et nuit), photopériode de $16 \mathrm{~h}$ assurée par des ampoules incandescentes (150W à tous les 2,2 $\mathrm{m}$ et à environ $1,0 \mathrm{~m}$ audessus des plants), irrigation à l'aide d'une rampe d'aspersion mobile pour maintenir le substrat à au moins 70 p. 100 de la saturation effective (irrigation déterminée par la pesée de plateaux échantillons), et fertilisation hebdomadaire de plantules, âgées d'un mois ou plus, à l'aide d'un engrais soluble, le 20-20-20 (Plant Product Co.) à une concentration en p.p.m. de $360: 114: 215$ (N:P:K), incluant 80 p.p.m. de $\mathrm{Mg}^{+2}$ sous forme de $\mathrm{MgSO}_{4} \cdot 7 \mathrm{H}_{2} \mathrm{O}$. Une fois placés sous ombrière, les plants furent fertilisés manuellement et seulement avec du 10-52-10 (Plant Product Co.), une fois par semaine à une concentration en p.p.m. de 130: 297: 107 (N:P:K).

L'ombrière était une structure fabriquée de tuyaux galvanisés (diamètre: $2,54 \mathrm{~cm}$ ), recouverte d'une toile à ombrager fabriquée de matière plastique, verte, tissée et pouvant réduire l'intensité lumineuse de 57 p. 100. La toile était fixée à la structure métallique au début du mois de mai et enlevée à la fin d'octobre.

La croissance en hauteur et le diamètre au collet des plants furent mesurés et le rapport hauteur-diamètre (H/D) moyen, appellé aussi indice de robustesse, fut calculé à partir du 25 mai jusqu'au 28 septembre 1981. En général, on a pris en considération, lors de l'évaluation des plants, les critères d'acceptation des plants de l'époque (1980) et des dernières années $(1984,1985)$ préparés et utilisés par le MER (table 2). L'acclimatation minimale d'un mois suggéré par le MER en 1980 et 1984 devait se faire généralement sous ombrière pour endurcir les plants et empêcher l'insolation ou la dessiccation par le vent et le froid du matériel à planter.

Les analyses statistiques effectuées ici sur des mesures et des calculs étaient en fait des analyses de variance pour des dispositifs complètement aléatoires. Chaque analyse fut suivie d'un test de Duncan afin de déterminer les différences significatives entre les moyennes (Steel et Torrie 1980).

\section{Résultats et discussion}

La croissance en hauteur des plants de sept séries, ensemencés à des dates différentes et dont les durées de culture sous serre et ombrière variaient, est représentée graphiquement à la figure 1. Les plants qui n'ont pu recevoir quatre semaines d'acclimatation sous ombrière, tel que dicté par les critères d'acceptation du MER datés de 1980 et 1984 , ou qui n'ont pu atteindre $15,0 \mathrm{~cm}$ de hauteur ont été classés inacceptables. Les traitements ont été classés acceptables lorsque les plants étaient acclimatés et d'une hauteur moyenne égale ou supérieure à $15,0 \mathrm{~cm}$.

Les traitements permettant l'obtention de plants acclimatés et d'une hauteur acceptable comprenaient une durée de culture (serre + ombrière) minimale de 21 semaines lorsque l'ensemencement s'effectuait le 9 février et de 18 semaines lorsqu'il avait lieu entre le 2 mars et le 25 mai. La croissance en hauteur des plants ensemencés à la mi-juin était quant à elle trop faible. Généralement, plus la durée de culture sous serre était longue, plus la croissance en hauteur se prolongeait. Les diminutions de hauteur des plants des traitements $30 / 3$ (série 1), $24 / 3$ (série 3) et $24 / 0$ (série 4) ont possiblement été causées par les différentes positions des plateaux sur les tables de la serre. Les résultats de notre étude s'accordent bien avec les travaux de Léger (1980), Barteaux et Kreiberg (1982), Kelly (1982), Carlson (1983) et Langlois (1985) qui ont décrit des plants de cultures hivernales âgés de 21 semaines ou plus lors de la plantation.

\begin{tabular}{|c|c|c|c|c|c|c|c|}
\hline \multirow{2}{*}{$\begin{array}{l}\text { No } \\
\text { de série } \\
\text { des plants }\end{array}$} & \multirow{2}{*}{$\begin{array}{l}\text { Date } \\
\text { d'ensemencement }\end{array}$} & \multirow{2}{*}{$\begin{array}{l}\text { Nombre de } \\
\text { plateaux } \\
\text { ensemencés }\end{array}$} & \multicolumn{5}{|c|}{ Date de la collecte des données } \\
\hline & & & mai & juin & juillet & août & septembre \\
\hline 1 & 9 février & 14 & $\begin{array}{c}25 \\
(15 \text { sem. })^{*}\end{array}$ & $\begin{array}{c}15 \\
(18 \mathrm{sem} .)\end{array}$ & $\begin{array}{c}6,27 \\
(21,24 \text { sem. })\end{array}$ & $\begin{array}{c}17 \\
\text { (27 sem.) }\end{array}$ & $\begin{array}{c}7,28 \\
(30,33 \text { sem. })\end{array}$ \\
\hline 2 & 2 mars & 14 & $\begin{array}{c}25 \\
\text { (12 sem.) }\end{array}$ & $\begin{array}{c}15 \\
\text { (15 sem.) }\end{array}$ & $\begin{array}{c}6,27 \\
(18,21 \text { sem. })\end{array}$ & $\begin{array}{c}17 \\
\text { (24 sem.) }\end{array}$ & $\begin{array}{c}7,28 \\
(27,30 \text { sem. })\end{array}$ \\
\hline 3 & 23 mars & 14 & $\begin{array}{c}25 \\
(9 \text { sem. }\end{array}$ & $\begin{array}{c}15 \\
(12 \text { sem.) }\end{array}$ & $\begin{array}{c}6,27 \\
(15,18 \text { sem. })\end{array}$ & $\begin{array}{c}17 \\
(21 \mathrm{sem} .)\end{array}$ & $\begin{array}{c}7,28 \\
(24,27 \text { sem. })\end{array}$ \\
\hline 4 & 13 avril & 14 & $\begin{array}{c}25 \\
\text { (6 sem.) }\end{array}$ & $\begin{array}{c}15 \\
\text { (9 sem.) }\end{array}$ & $\begin{array}{c}6,27 \\
(12,15 \mathrm{sem} .)\end{array}$ & $\begin{array}{c}17 \\
\text { (18 sem.) }\end{array}$ & $\begin{array}{c}7,28 \\
(21,24 \text { sem. })\end{array}$ \\
\hline 5 & 4 mai & 12 & & $\begin{array}{c}15 \\
\text { (6 sem.) }\end{array}$ & $\begin{array}{c}6,27 \\
(9,12 \text { sem.) }\end{array}$ & $\begin{array}{c}17 \\
(15 \text { sem.) }\end{array}$ & $\begin{array}{c}7,28 \\
(18,21 \text { sem. })\end{array}$ \\
\hline 6 & 25 mai & 10 & & & $\begin{array}{c}6,27 \\
(6,9 \text { sem. })\end{array}$ & $\begin{array}{c}17 \\
\text { (12 sem.) }\end{array}$ & $\begin{array}{c}7,28 \\
(15,18 \text { sem. })\end{array}$ \\
\hline 7 & 15 juin & 8 & & & $\begin{array}{c}27 \\
\text { (6 sem.) }\end{array}$ & $\begin{array}{c}17 \\
\text { (9 sem.) }\end{array}$ & $\begin{array}{c}7,28 \\
(12,15 \mathrm{sem} .)\end{array}$ \\
\hline
\end{tabular}

-Äge des plants. 
Tableau 2. Critères d'acceptation des plants d'épinette noire cultivés en conteneurs.

\begin{tabular}{|c|c|c|c|c|}
\hline \multirow[b]{2}{*}{ Caractéristiques } & \multicolumn{4}{|c|}{ Remarques } \\
\hline & MER, 1980 & MER, 1984 & MER, 1985 & GIROUARD \\
\hline Hauteur $(\mathrm{H})$ moyenne minimale & $15,0 \mathrm{~cm}$ & $12,5 \mathrm{~cm}$ & $15,0 \mathrm{~cm}$ & $15,0 \mathrm{~cm}$ \\
\hline Diamètre (D) moyen minimal au collet & $2,5 \mathrm{~mm}$ & $1,5 \mathrm{~mm}$ & $1,8 \mathrm{~mm}$ & $2,0 \mathrm{~mm}$ \\
\hline $\begin{array}{l}\text { Rapport hauteur-diamètre (H/D) moyen } \\
\text { maximal }\end{array}$ & 6,0 & 10,0 & - & 9,0 \\
\hline $\begin{array}{l}\text { Rapport tige-racines (masses anhydres) } \\
\text { moyen maximal }\end{array}$ & 3,0 & - & - & - \\
\hline $\begin{array}{l}\text { Indice de qualité morphologique (Dickson) } \\
\text { moyen minimal }\end{array}$ & 0,10 & - & - & - \\
\hline Etat du matériel & $\begin{array}{l}\text { Libre de maladies et } \\
\text { de déficiences } \\
\text { minérales }\end{array}$ & $\begin{array}{l}\text { Libre d'insectes, de } \\
\text { maladies, de déficiences } \\
\text { minérales, de dessiccation, } \\
\text { de tiges brisées ou } \\
\text { malformées; mottes } \\
\text { extraites non brisées. }\end{array}$ & $\begin{array}{l}\text { Libre d'insectes, de } \\
\text { déficiences minérale } \\
\text { sur plus de la moitié } \\
\text { tige, de tiges malform } \\
\text { pousses latérales plu } \\
\text { deux tiers de la haute } \\
\text { mottes extraites non }\end{array}$ & $\begin{array}{l}\text { s, de } \\
\text { essiccation } \\
\text { auteur de la } \\
\text { brisées, de } \\
\text { les que les } \\
\text { lant, des }\end{array}$ \\
\hline Acclimatation minimale & 1 mois & 1 mois & - & 1 mois \\
\hline Tolérance maximale de plants rejetés & $10 \%$ & $25 \%$ & $15 \%$ & $15 \%$ \\
\hline
\end{tabular}

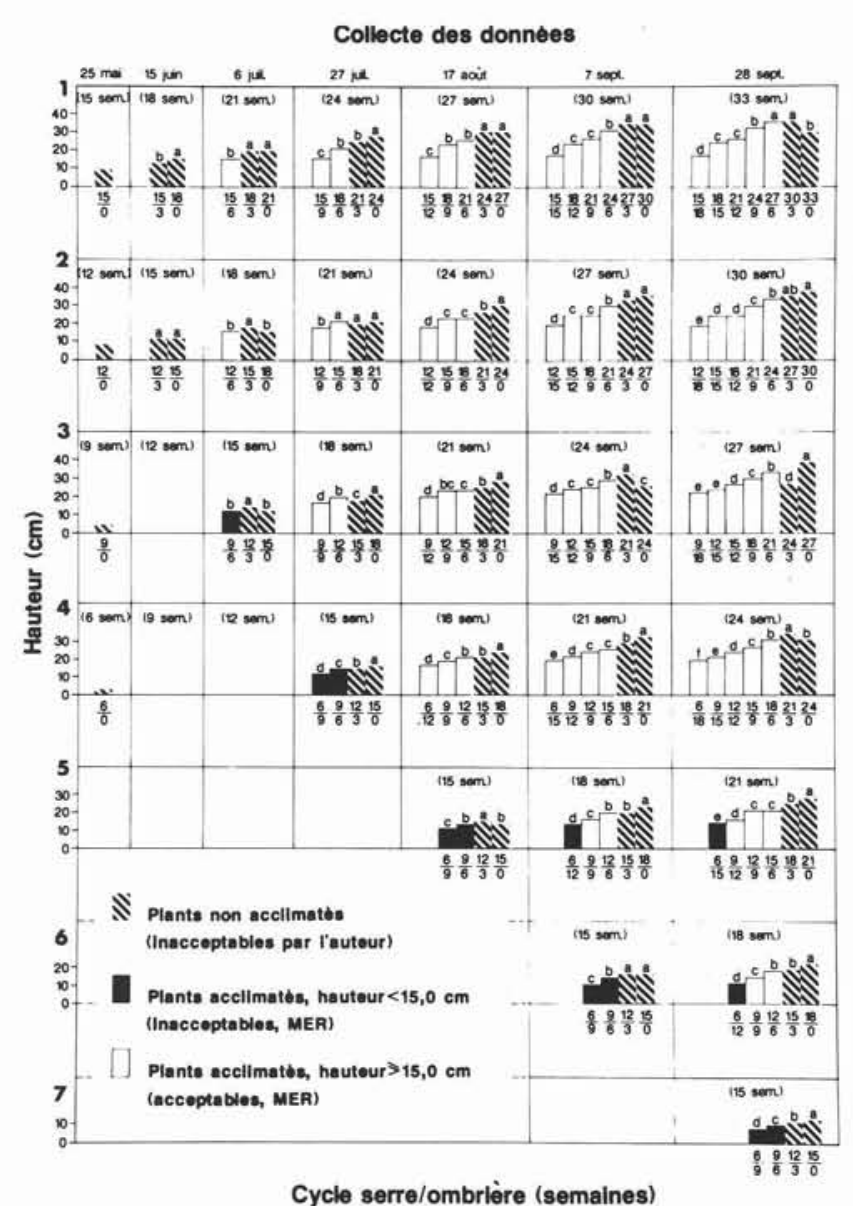

Figure 1. Hauteur des plants de sept dates d'ensemencement: $1=9$ fév. $2=2$ mars, $3=23$ mars, $4=13$ avril, $5=4$ mai, $6=25$ mai, $7=15$ juin. Le nombre de semaines entre parenthèses désigne l'âge des plants. Le quotient ou cycle serreombrière indique la durée de culture sous serre suivie de la durée sous ombrière. "Les moyennes des plants du même âge d'une série portant la même lettre ne sont pas significativement différentes au seuil $P=0,05$.
La figure 2 montre seulement quelques traitements où les plants acclimatés avaient un diamètre moyen au collet qui était inférieur à $1,8 \mathrm{~mm}$. Si on rend le critère d'acceptation du diamètre plus exigeant en l'augmentant à $2,0 \mathrm{~mm}$, tel que suggéré par Girouard (1985) pour le pin gris, les semis de trois autres traitements (cycle $9 / 6$ des séries 4,5 et 6 ) seraient rejetés. On remarque que les plants des trois premières séries, une fois âgés de 24 semaines ou plus, ont un diamètre au collet de $3,0 \mathrm{~mm}$ et plus. Aussi, on note des diminutions de diamètre au collet des plants des traitements $27 / 6$ et $33 / 0$ (série 1), 27/3 (série 2), 24/3 (série 3) et 21/3 (série 4). Ces diminutions ont peut-être été causées par la position du matériel sur les tables. Des diamètres de tiges variant de 2 à $3 \mathrm{~mm}$, selon l'âge des plants, ont déjà été relevés par Léger (1980), Krause (1982), Carlson (1983) et Langlois (1985).

En ce qui a trait à l'indice de robustesse (le rapport hauteur-diamètre ou $H / D$ ), qui révèle la nature trapue ou grêle des semis, seuls les plants d'un traitement de chacune des quatre premières séries ont dépassé la valeur acceptable, c'est-à-dire 10, selon les critères du MER de 1984 (figure 3). Le matériel rejeté avait été acclimaté pendant six semaines, à partir du 17 août après avoir été cultivé le plus longtemps possible sous serre. Lorsque l'acclimatation était d'une durée de neuf semaines et commençait le 27 juillet, l'indice de robustesse était toujours de 10 ou moins. Bien que Roller (1977) ait suggéré que l'indice de robustesse des plants d'épinette noire ne devrait pas excéder une valeur de 6 , sans quoi il y a risque que le matériel soit endommagé par le vent, une sécheresse ou même le gel, des auteurs (Girouard 1982, Krause 1982 et Langlois 1985) ont publiés récemment des données où le rapport hauteur-diamètre variait de 7 à 10. Ces auteurs ne semblaient pas être inquiétés par des rapports plus élevés. Toutefois, aucune preuve n'était fournie justifiant l'acceptation d'un rapport plus grand que 6 .

Il importe de souligner que les premiers plants acclimatés dont la hauteur des tiges, le diamètre au collet et la robustesse étaient acceptables furent mis sous ombrière le 25 mai, au moment où ils étaient âgés de 15 semaines. Les plants étaient acclimatés et prêts à livrer aux reboiseurs six semaines plus tard, c'est-à-dire le 6 juillet. 


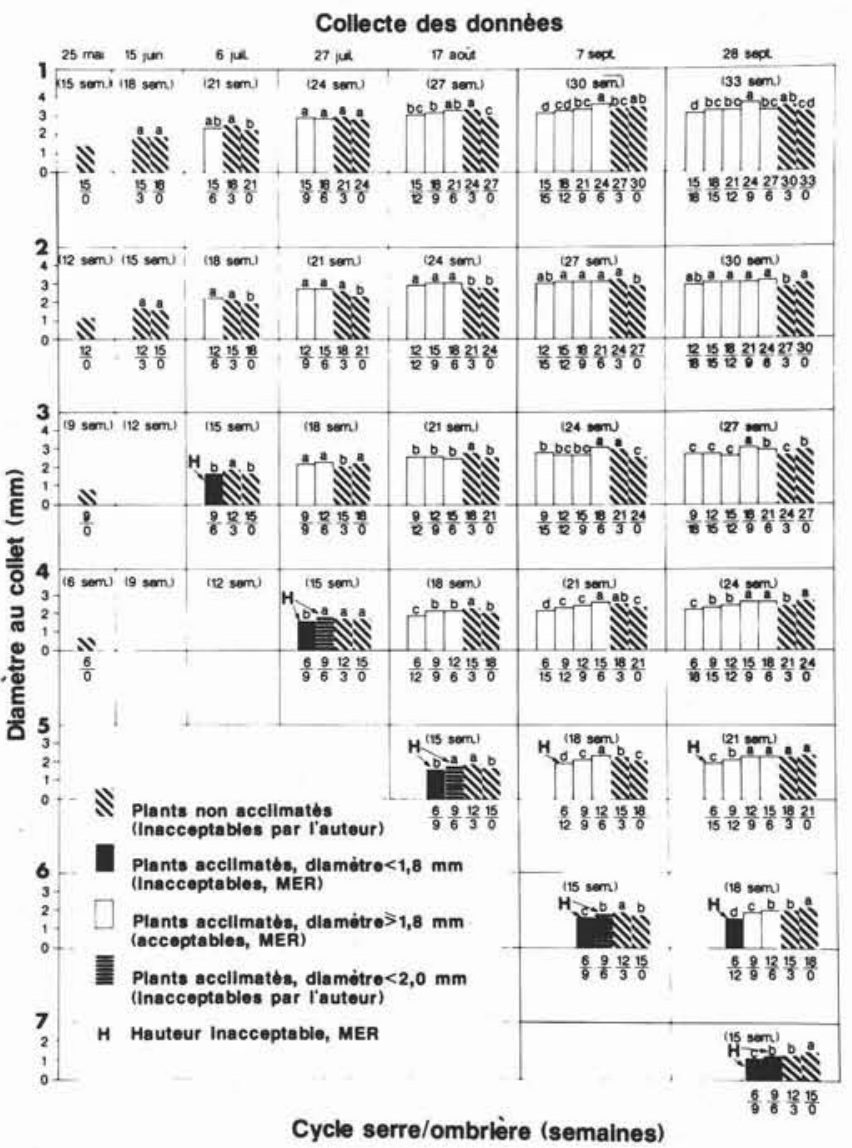

Figure 2. Diamètre au collet des plants de sept dates d'ensemencement: $1=9$ fév., $2=2$ mars, $3=23$ mars, $4=13$ avril, $5=4$ mai, $6=25$ mai, $7=15$ juin. Le nombre de semaines entre parenthèses indique l'âge des plants. Le quotient ou cycle serre-ombrière indique la durée de culture sous serre suivie de la durée sous ombrière. "Les moyennes des plants du même âge d'une série portant la même lettre ne sont pas significativement différentes au seuil $P=0,05$.

Si la serre avait pu contenir seulement des plants devant sortir le 25 mai, l'ensemencement d'une deuxième culture à cette date aurait permis d'obtenir des plants acceptables comme dans le cas de la série 6. La sortie de serre des plants aurait pu se faire le 27 juillet (cycle 9/9) ou le 17 août (cycle 12/6), c'est-à-dire 76 ou 55 jours avant le premier gel au sol (le 11 octobre) et quand la photopériode était de 15.1 ou $14.2 \mathrm{~h}$ (Russelo et al. 1974). Cependant, lorsque les plants de la deuxième culture étaient prêts à sortir de la pépinière (le 28 septembre), aucune garantie ne pouvait être fournie en ce qui concerne la résistance des plants aux basses températures d'automne et d'hiver.

En 1983, Carlson avait suggéré que les plants aient entre 28 et 42 jours pour s'endurcir avant le repiquage ou le début de la période d'hivernage. Colombo (1984) avait recommandé, pour la pépinière de Swastika ( $48^{\circ}$ de latitude nord), en Ontario, que la sortie des plants de la serre se fasse seulement après l'initiation des bourgeons, c'est-à-dire le 14 août, Iorsque la photopériode est de $14.4 \mathrm{~h}$; ensuite, que l'on fournisse aux plants un minimum de 42 jours d'acclimatation à l'extérieur pour permettre à ces derniers de résister à des températures de -5 à $-8^{\circ} \mathrm{C}$. De même, D'Aoust et Trudel (1985) ont montré qu'un mois en ombrière, à la fin d'août,

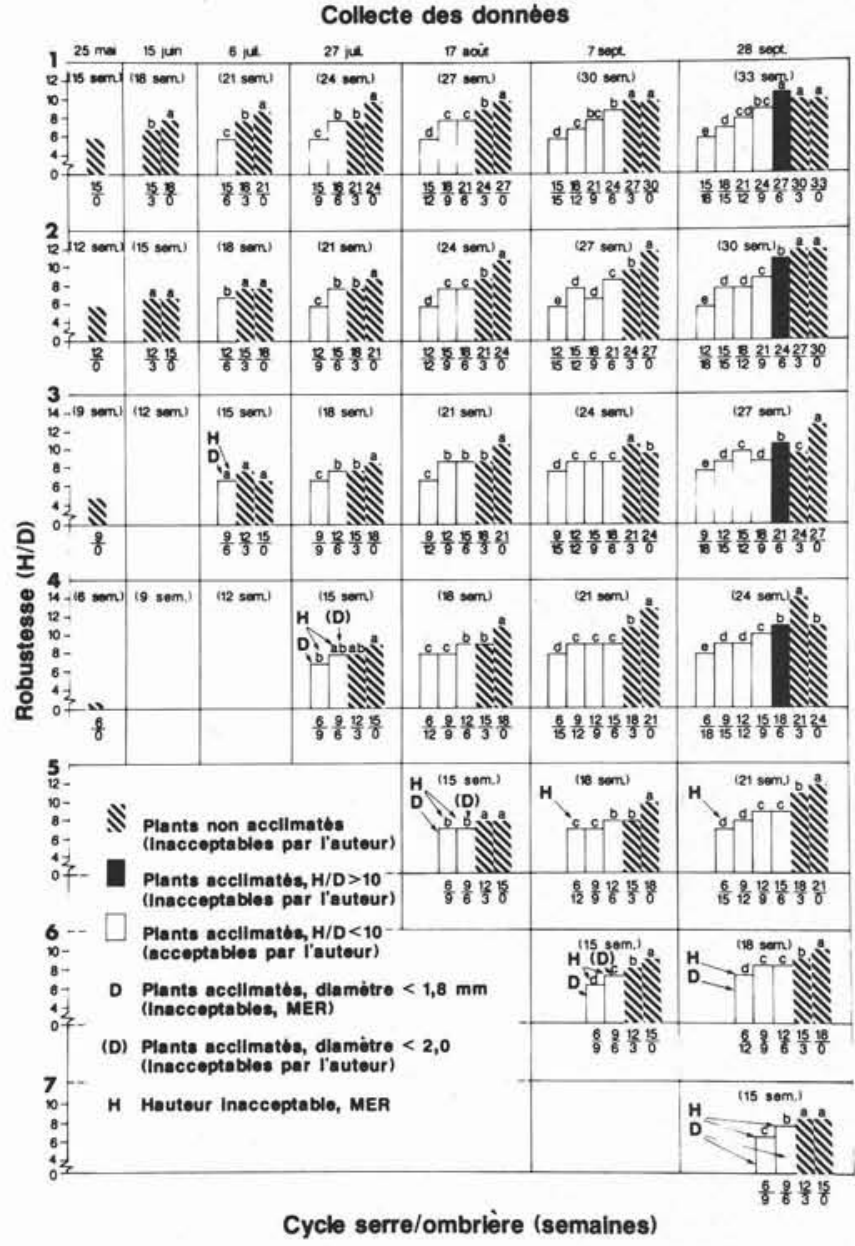

Figure 3. Robustesse (H/D) des plants de sept dates d'ensemencement: $1=9$ fév., $2=2$ mars, $3=23$ mars, $4=13$ avril, $5=4$ mai, $6=25$ mai, $7=15$ juin. Le nombre de semaines entre parenthèses désigne l'âge des plants. Le quotient ou cycle serre-ombrière indique la durée de culture sous serre suivie de la durée sous ombrière. "Les moyennes des plants du même âge d'une série portant la même lettre ne sont pas significativement différentes au seuil $P=0,05$.

permet aux semis d'épinette noire de s'acclimater. Miron (1985) avait indiqué qu'il fallait commencer à mettre les plants en dormance un mois avant les premiers gels d'automne et sortir les plants à l'extérieur sur le site d'hivernage dès que l'entrée en dormance était bien induite. De plus, il fallait que le bourgeon terminal des plants soit bien formé, bien évident et de couleur brune au moment des premiers gels.

\section{Conclusion}

L'idée de produire des cultures multiples de plants d'épinette noire au Québec à l'aide de serres et d'ombrières est intéressante, mais lorsque l'on prend en considération les critères d'acceptation des plants utilisés par le MER et le fait que les serres sont chauffées le moins longtemps possible, on se rend compte assez rapidement que le terme "cultures multiples" se limite à deux cultures par année surtout à Sainte-Foy.

Par exemple, on peut commencer avec un ensemencement au début de février ou mars, laisser les plants sous serre jusqu'au 25 mai et ensuite les mettre sous l'abri d'une 
ombrière où la croissance peut continuer et l'acclimatation se faire. L'ensemencement d'une deuxième culture sous serre dès le 25 mai peut permettre l'obtention de plants acceptables à condition que la sortie de serre ne se fasse pas plus tard que le 17 août.

\section{Remerciements}

Nous tenons à remercier M. Roger Mongrain dont la collaboration durant cette étude nous aura été très précieuse. Des remerciements sincères s'adressent aux réviseurs et à tous ceux qui ont contribué à la réalisation du manuscrit.

\section{Références}

Barbulescu, A. 1982. An integrated industrial system for the production of tree seedling containers. In Proc. Can. Containerized Tree Seedling Symp. Édité par J.B. Scarratt, C. Glerum et C.A. Plexman. Can. For. Serv. et Ont. Min. Nat. Resour. COJFRC Symp. Proc. O-P-10, Toronto, Ont., 14-16 sep. 1981. pp. 225-229.

Barteaux, M.K. et N.H. Kreiberg. 1982. The status of container planting in Canada. 7. New Brunswick. In Proc. Can. Containerized Tree Seedling Symp. Édité par J.B. Scarratt, C. Glerum et C.A. Plexman. Can. For. Serv. et Ont. Min. Nat. Resour. COJFRC Symp. Proc. O-P-10, Toronto, Ont., 14-16 sep. 1981.pp. 45-48.

Carlson, L.W. 1983. Conseils pour la culture en récipients des semis de conifères dans les provinces des Prairies. Minist. Environ. Can., Serv. can. for., Cent. rech. for. Nord, Edmonton, Alberta. NOR-X-214 F

Clark, P.A. 1983. New Brunswick Natural Resources. What are we presently producing at provincial forest nursery? In Proc. Stock Quality Workshop. Édité par R.D. Hallett et M.D. Cameron. Can. For. Ser., Marit. For. Res. Cent., Workshop Proc. No. 1, Fredericton, N.B. 9 mars 1982. pp. 29-30.

Colombo, S.J. 1984. Acclimatizing and overwintering spruce container stock. In Proc. Nurserymen's Meeting, Ont. Min. Nat. Resour., Kemptville, Ont., 13-17 juin 1983. pp. 79-81.

D'Aoust, A.L. et D. Trudel. 1985. L'endurcissement au gel et au stress du milieu, par des traitements photo-thermopériodiques, chez des semis d'épinette noire en conteneurs. In Deuxième atelier de travail sur la culture des semis en récipients. Serv. can. for., Cent. rech. for. Laurentides; Minist. Energie Ress. Qué.; et Ordre des Ing. For. Qué., Sainte-Foy, Qué. 29-30 nov. 1984. pp. 76-85.

Deschênes, E. et W. Whalen. 1983. Fraser Inc. (Présentation). In Proc. Stock Quality Workshop. Édité par R.D. Hallett et M.D. Cameron. Can. For. Serv., Marit. For. Res. Cent., Workshop Proc. No. 1, Fredericton, N.B., 9 mars 1982. pp. 29-30.

Forcier, L. 1980. La culture des semis en récipients en Ontario. In Atelier de travail sur la culture des semis en récipients. Serv. can. for., Cent. rech. for. Laurentides; Minist. Energie Ress. Qué.; et Ordre Ing. For. Qué., Québec, Qué. 18-20 mars 1980. pp. $156-177$.

Girouard, R.M. 1982. Production en serre de plants d'épinette blanche, d'épinette noire, de pin gris et de pin rouge dans trois types de conteneurs. Minist. Environ. Can., Serv. can. for., Cent. rech, for. Laurentides, Sainte-Foy, Qué, Rapp. d'inf. LAU-X-57.

Girouard, R.M. 1985. Cultures multiples de plants de pin gris sous serre et ombrière. In Deuxième atelier de travail sur la culture des semis en récipients. Serv. Can. for., Cent. rech. for. Laurentides; Minist. Energie Ress. Qué.; et Ordre Ing. For. Qué., Sainte-Foy, Qué. 29-30 nov. 1984. pp. 86-96.
Gonzalez, A. 1981. Bref aperçu des propriétés chimiques et physicochimiques de la tourbe utilisée dans les conteneurs du Québec (CRIQ). Minist. Environ. Can., Serv. can. for., Cent. rech. for. Laurentides, Sainte-Foy, Qué. LAU-X-48.

Kay, W.C. 1973. Greenhouse production of conifer seedlings. Papier présenté lors d'une réunion, American Society of Agricultural Engineers, Chicago, Illinois, 11-14 déc. 1973, 8 p.

Kelly, G. 1982. The status of container planting programs in Canada 9. Prince Edward Island. In Proc. Can. Containerized Tree Seedling Symp. Édité par J.B. Scarratt, C. Glerum et C.A Plexman. Can. For. Serv. et Ont. Min. Nat. Resour., COJFRC Symp. Proc. O-P-10, Toronto, Ont., 14-16 sep. 1981. pp. 51-54.

Krause, H.H. 1982. Early growth of bare-root and paperpot plantations at various locations in New Brunswick. In Proc. Can. Containerized Tree Seedling Symp. Édité par J.B. Scarratt, C. Glerum et C.A. Plexman. Can. For. Serv. et Ont. Min Nat. Resour., COJFRC Symp. Proc. O-P-10, Toronto, Ont., 1416 sep. 1981. pp. 355-364.

Langlois, C.-G. 1985. La culture en récipients - croissance, conditions culturales et fertilisation. In Deuxième atelier de travail sur la culture des semis en récipients. Serv. can. for., Cent. rech. for. Laurentides; Minist. Energie Ress. Qué.; e Ordre Ing. For. Qué., Sainte-Foy, Qué. 29-30 nov. 1984. pp. 3664.

Léger, L. 1980. Notes sur les plants en récipients au NouveauBrunswick. In Atelier de travail sur la culture des semis en récipients. Serv, can. for., Cent. rech. for. Laurentides; Minist. Energie Ress. Qué.; et Ordre Ing. For. Qué., Québec, Qué. 1820 mars 1980 . pp. $178-183$.

McNeish, S. et W.D.F. Heinstein. 1983. NBIP Limited. (Présentation) In Proc. Stock Quality Workshop. Édité par R.D. Hallett et M.D. Cameron. Can. For. Serv., Marit. For. Res. Cent., Workshop Proc. No. 1, Fredericton, N.B., 9 mars 1982. pp. 10-16.

Miron, F. 1985. La lutte contre le froid et l'hivernement des plants en récipients. In Deuxième atelier de travail sur la culture des semis en récipients. Serv. can. for., Cent. rech. for. Laurentides: Minist. Energie Ress. Qué., et Ordre Ing. For. Qué., Sainte-Foy, Qué. 29-30 nov. 1984. pp. 97-104.

Myers, J.F. 1979. Greenhouse cropping and container washing. Proc. Intermountain Nurserymen's Assoc. Meeting, Aspen, Colorado. 13-16 août 1979. pp. 53-55.

Owston, P.W. 1974. Two-crop production of western conifers. In Proc. North Am. Containerized For. Tree Seedlings Symp. Great Plains Agric. Counc. Publ. 68. pp. 104-111.

Paillé, G. et W.D.F. Brown. 1980. La culture des semis en récipients chez C.I.P. In Atelier de travail sur la culture des semis en récipients. Serv. can. for., Cent. rech. for. Laurentides; Minist. Energie Ress. Qué.; et Ordre Ing. For. Qué., Québec, Qué. 1820 mars 1980. pp. 114-134.

Roller, K.J. 1977. Suggested minimum standards for containerized seedlings in Nova Scotia. Can. Dep. Environ., Can. For. Serv. Marit. For. Res. Cent., Fredericton, N.B. M-X-69.

Russelo, D., S. Edey et J. Godfrey. 1974. Selected tables and conversions used in agrometeorology and related fields. Can. Dep. Agric., Pub. 1522.

Schabas, W. 1978. A third of a billion trees by the year 2000 from containerized spruce seedlings. Pulp \& Paper Canada 79(12): $52-54$

Steel, R.G.D. et J.H. Torrie. 1980. Principles and Procedures of Statistics. McGraw-Hill, Toronto.

Zalasky, H. 1983. Optimizing containerized conifer seedling production in the Prairie Region. Can. Dep. Environ., Can. For. Serv., North. For. Cent. Edmonton, Alberta. For. Manage. Note 21. 